\title{
The University of Pennsylvania RoboCup Legged Soccer Team
}

\author{
Sachin Chitta, William Sacks, Jim Ostrowski, Aveek Das, and P.K. Mishra \\ University of Pennsylvania
}

\section{Introduction}

This paper describes the University of Pennsylvania's RoboCup 2001 Legged League team, called the UPennalizers. This year's team was very successful in both the main competition, placing third out of sixteen teams, and the RoboCup Technical Challenges, where we placed second. Much of this success can be credited to our being able to build on a strong foundation, in both software architecture and low-level, fast locomotion, developed last year. We also were one of only two teams to utilize sound communication between robots, which gave a significant advantage in resolving conflicts between team members.

\section{Team Development}

Team Leader: James P. Ostrowski

- Department of Mechanical Engineering and Applied Mechanics, University of Pennsylvania, United States

- Assistant Professor, attended the competition

\section{Team Members:}

Sachin Chitta and Aveek K. Das

- Department of Mechanical Engineering and Applied Mechanics, University of Pennsylvania, United States

- Graduate Students, attended the competition

William Sacks

- Williams College

- Undergraduate junior, attended the competition

Caroline Klose, P.K. Mishra

- Computer and Information Science, University of Pennsylvania, U.S.

- Undergraduate Senior and Master's student, did not attend

Web page http://www.cis.upenn.edu/robocup/

\section{Architecture}

In 3] we presented our hierarchical structure for control of autonomous robots. This architecture is compact and can be applied across multiple platforms. Figure 1] shows the top level overview, along with a schematic of the main logical sensing and actuation blocks that are used. The major blocks are the planner, the extended logical sensor module(XLSM) and the extended logical actuator module (XLAM). 

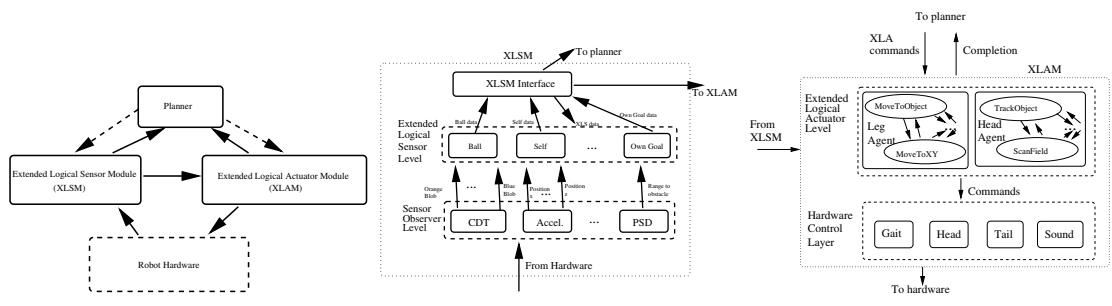

Fig. 1. (left) Top-level view of overall structure. Arrows indicate data flow; dashed arrows indicate commands. (center) XLSM (right) XLAM

Extended logical sensor module (XLSM) A logical sensor is defined as a software construct that converts raw sensory data into some application domain information. An extended logical sensor (XLS), then, is a sensory agent capable of modifying its operation (mode-switching) based on state information. An example would be an XLS for tracking a moving object in a noisy environment using vision. If the object can be seen, its position is recorded. If the object is occluded, the XLS can use Kalman filtering to give a best estimate of the object's position. If a second sensor were added to the system (radar for example), the XLS would be modified to use both sensors (or prediction) to detect the target. Decision making agents (planners) are only aware that they have access to a "sensor" that reads target position.

Extended logical actuator module (XLAM) A logical actuator is defined as the output counterpart to a logical sensor. It converts application domain commands into physical actuator outputs. An extended logical actuator (XLA) is an agent capable of performing some low-level "inner loop" control on physical actuators. XLA primitives for soccer might include moving to a position in the field, moving toward an object, kicking an object, etc. Planners need not be aware of operations and controllers that are involved in achieving these tasks. In fact, the operations are the same for a legged robot as for a wheeled robot-so the planner need not even know how the task is accomplished.

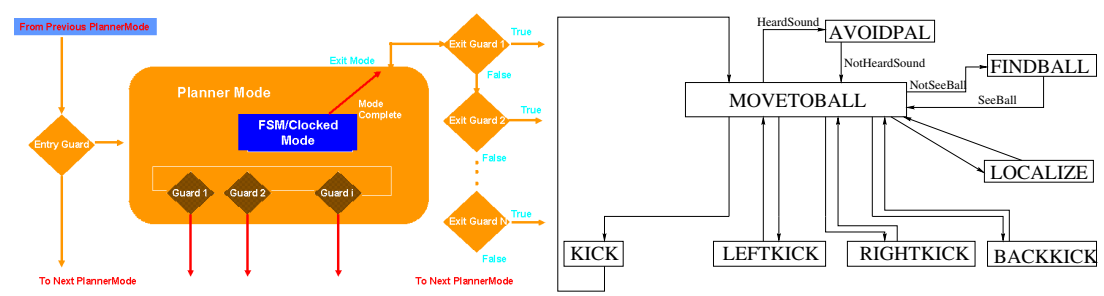

Fig. 2. Switching between Planner Modes (left) and a sample FSM used for generation of Plannertask table in example (right) 
Planner The Planner is the decision making module of the robot and forms the highest level in the hierarchy. The main decision making structure of the planner is the Plannertask table, which represents the strategy for a robotic soccer player in a compact form. This formalism fits well within a hybrid systems approach, where low-level sensing and dynamics have been abstracted into higher-level, discrete modes. The left diagram in Figure 2 shows an example flow of a table, where a transition to a new mode results first in an entry guard being checked. If the guard condition is true, a new, mixed finite state machine and clocked, feedback loop is initiated (a "mode"). During the its execution, any number of guards may be checked to see if a mode switch is called for. If not, then at the completion of the mode a set of exit guards are used to determine the next mode.

A simple example is shown in Figure 2 of a PlannerTask table structure. The finite state machine executes the simple task of approaching and kicking a ball. The type of kick, e.g., RIGHTKICK, BACKKICK, (forward) KICK, is chosen depending on the player's and ball's location on the field. For example, if the robot is facing the wrong way it executes a back kick. As shown Figure 2, transitions between modes are selected by clocked guards, such as SeeBall, and mode completions, such as the transition from MoveToBall to a kick.

\section{Omnidirectional Walking Capability}

The robots have been programmed to have an omnidirectional walking capability. The XLA modes need to specify only the required translational and rotational components of velocity for the robot. These are then converted, using inverse kinematics, to required trajectories for the foot placement point of each foot. The robots use a trot walking gait which means that diagonally opposite pairs of legs move together. The foot placement point (which is the point of the foot that comes into contact with the ground) goes through a rectangular trajectory as it comes into and out of contact with the ground. The velocity of the robot is assumed to remain constant for each step. Thus, changes in velocity are initiated only at the end of a step. The duty factor for the gait is 0.5 which means that each foot of the robot spends as much time out of contact with the ground as it does in contact with the ground. This gait gives faster speeds and smoother transitions during changes in velocity. More details are in [1].

\section{$5 \quad$ Pal and Foe Detection}

The strategy for the game requires keeping track of all other players. This is complicated by the fact that the robots are not uniformly covered by a single color, but instead have multiple stickers distributed over the body. After sorting the blobs by team color and size, the largest blob is chosen to represent a particular player, and then other blobs are compared to see whether they belong to the same player. If the distance to the next largest blob is within a certain threshold (we have chosen this to be less than three times the length of the largest blob), the smaller blob is assigned to the same player as the larger blob. 
This process is continued for all blobs representing the player types. The factor of three is conservative, sometimes merging two players that are near each other. This is preferable to a more aggressive approach where "ghost" players could be spuriously detected.

Keeping track of pals and foes The observer must next determine which player in memory corresponds to each player in the current view. Our algorithm for matching is similar to one presented by Veloso et al. [4]. For a player A in the current view and a player B in memory (with B on the same team as $\mathrm{A}$ ), the likelihood that $\mathrm{A}$ and $\mathrm{B}$ are the same player is calculated as: likelihood $(A, B)=$ $\left(B\right.$ 's uncertainty) $* \frac{1}{(d(A, B))^{3}} * \frac{1}{(\Delta \theta(A, B))}$. Uncertainty ranges from 0.5 (recently seen) to 100 (not seen in a long time). Thus, it is more likely that A and B are the same player if B has not been seen in a while, and also if A and B are close. Then, for each player $A$ in the current view, the player B in memory that gives the highest likelihood $(\mathrm{A}, \mathrm{B})$ is replaced in memory by $\mathrm{A}$.

\section{Potential Field Approach}

We have also utilized a potential field approach (see, e.g., Khatib [2]) to obstacle avoidance. In our situation, the goal exerts an attractive force on the robot, $F_{\text {goal }}=-k_{1} *\left(x-x_{\text {goal }}\right)$, where $x$ and $x_{\text {goal }}$ are the positions of the robot and goal, and $k_{1}$ is a positive constant; while obstacles exert repulsive forces

$$
F_{\text {obstacle }}=k_{2} *\left(\frac{1}{\left(x-x_{\text {obstacle }}\right)}-\frac{1}{x_{0}}\right) * \frac{1}{\left(x-x_{\text {obstacle }}\right)^{2}} * \nabla\left(x-x_{\text {obstacle }}\right),
$$

where $x$ and $x_{\text {obstacle }}$ are positions of the robot and obstacle, $x_{0}$ is the maximum distance at which an obstacle exerts a force, and $k_{2}$ is a positive constant.

Summing the forces on the robot gives the total force vector; the robot then moves in the direction of this vector. This effectively steers the robot toward the goal and away from the obstacles. A major advantage of this method is that it allows real-time planning, because the calculations at any point are not computationally expensive. Some important modifications had to be made before using this technique. When the obstacles were very far away they still exerted a repulsive force. As mentioned in [2], preventing forces from such obstacles leads to a more stable path. Hence, the force from obstacles further than a certain fixed distance was ignored. Similarly, obstacles behind the robot are ignored.

\section{Conclusion}

Contributions in RoboCup-2001 We felt our main contributions to RoboCup2001 were the full use of audio communication, a solid modular architecture (testing of many modules working together), and a very stable and fast dynamic trot gait based on inverse kinematics that is relatively easy to implement. 
Future work for RoboCup-2001 We will enter a team in next year's competition and will focus on three areas: 1) wireless communication; 2) decentralized control and estimation strategies; and 3) high-resolution image processing.

\section{References}

[1] S. Chitta and J. P. Ostrowski. New insights into quasi-static and dynamic omnidirectional quadruped walking. To appear in IEEE Int. Conf. Intelligent Robots and Systems, IROS01, October 2001.

[2] O. Khatib. Real-time obstacle avoidance for manipulators and mobile robots. International Journal of Robotic Research, 5(1):90-98, 1986.

[3] K. A. McIsaac, A. K. Das, J. M. Esposito, and J. P. Ostrowski. A hierarchical structure for hybrid control applied to AIBO robotic soccer. In Proc. IEEE/RSJ Intl. Conf. on Intelligent Robots and Systems (IROS), pages 1020-1025, Japan, November 2000.

[4] M. Veloso, M. Bowling, S. Achim, K. Han, and P. Stone. The CMUnited-98 champion small robot team. In M. Asada and H. Kitano, editors, RoboCup-98: Robot Soccer World Cup II, Berlin, 1999. Springer Verlag. 